\title{
Diagnóstico do conhecimento dos adolescentes sobre sexualidade
}

\author{
Diagnosis of knowledge on sexuality among adolescents \\ Diagnóstico de conocimientos de adolescentes sobre sexualidad \\ Sílvia Manuela Dias Tavares da Silva' ${ }^{1}$ ib https://orcid.org/0000-0003-4166-9803 \\ Maria Margarida da Silva Vieira Ferreira' ${ }^{1}$ io https://orcid.org/0000-0003-2232-7314 \\ Maria Manuela Amaral-Bastos ${ }^{1}$ is https://orcid.org/0000-0002-6217-7165 \\ Maria Amélia José Monteiro ${ }^{1}$ io https://orcid.org/0000-0002-47774-0554 \\ Germano Rodrigues Couto ${ }^{1}$ io https://orcid.org/0000-0002-5423-7375
}

Como citar:

Silva SM, Ferreira MM, Amaral-Bastos

MM, Monteiro MA, Couto GR. Diagnóstico

do conhecimento dos adolescentes sobre sexualidade. Acta Paul Enferm. 2020;33:eAPE20190210.

DOI

http://dx.doi.org/10.37689/ acta-ape/2020A00210

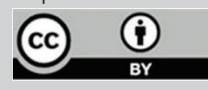

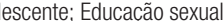
Enfermagem em saúde comunitária; Doenças sexualmente transmissíveis/prevenção e controle; Comportamento do adolescente

Keywords

Sexualitty; Adolescent;Sex education;Community health nursing; Sexually transmitted diseases/ prevention \& control; Adolescent behavior

Descriptores

Sexualidad; Adolescente; Educación sexual; Enfermería en salud comunitaria; Enfermedades de transmisión sexual/prevención \& control; Conducta del adolescente

Submetido 26 de Julho de 2019

Aceito 26 de Outubro de 2019

Autor correspondente

Sílvia Manuela Dias Tavares da Silva E-mail: sylviatavares@hotmail.com

\section{Resumo}

Objetivo: Realizar o diagnóstico do conhecimento dos adolescentes sobre sexualidade para a implementação, à posteriori, de um programa específico e direcionado de intervenção.

Métodos: Estudo observacional-descritivo, quantitativo, transversal, numa população de 250 alunos a frequentar o décimo ano. Aplicou-se um questionário com caraterização sociodemográfica e 0 Questionário de Conhecimentos sobre Sexualidade (QCS), constituído por 25 questões de resposta dicotómica (verdadeiro ou falso) e organizado em seis dimensões.

Resultados: Amostra de conveniência de 136 adolescentes, entre os 14-19 anos, maioritariamente do sexo masculino (54,4\%). A média de conhecimento é de 18,6 (DP=2,71), sendo as áreas em que os adolescentes apresentam menores conhecimentos as seguintes: "Primeira relação sexual e relações sexuais"; "Prevenção da gravidez"; e "Aconselhamento e atendimento em saúde sexual e reprodutiva". Existe apenas diferença significativa favorável às raparigas na dimensão "Prevenção da gravidez". Como fatores que influenciam positivamente o conhecimento dos adolescentes identificam-se a escolaridade ao nível do ensino superior dos pais com enfoque maior nas mães, e um dos pais ser profissional de saúde.

Conclusão: Constatou-se a necessidade de desenvolver um programa de intervenção direcionado à realidade da escola, incidindo nas áreas de "Primeira relação sexual e relações sexuais", "Prevenção da gravidez" e "Aconselhamento e atendimento em saúde sexual e reprodutiva", direcionando o programa para as diferenças do género, com especial relevância para os rapazes.

\section{Abstract}

Objectives: To diagnose adolescents' knowledge of sexuality for posterior implementation of a specific and targeted intervention program.

Methods: This was a quantitative-based cross-sectional (observational/descriptive) study including a population of 250 students enrolled in year ten of secondary education. A questionnaire for sociodemographic characterization was applied along with the Questionnaire on Sexuality Knowledge (QCS), which consisted of 25 questions in a dichotomous (true/false) answer scale separated into six dimensions.

Results: The convenience sample of 136 teenagers aged between 14 and 19 years. The majority of participants were men $(54.4 \%)$. Mean knowledge was $18.6(\mathrm{DP}=2.71)$; areas where adolescents showed the least knowledge are the following: "first sexual relation and sexual concerns"; "pregnancy prevention"; e "counseling and care in sexual and reproductive health". The only significant difference between dimensions was for "pregnancy prevention" (girls show higher knowledge). Factors positively impacting adolescents' knowledge are the parents' formal education level (higher education, especially for mothers) and one of the parents be a healthcare provider. 
Conclusion: The need to develop an intervention program targeting the reality of schools was identified, based on the areas of "first sexual relation and sexual concerns"; "pregnancy prevention"; and "counseling and care in sexual and reproductive health". The program should target gender differences and, especially, considering boys.

\section{Resumen}

Objetivo: Realizar un diagnóstico de conocimientos de los adolescentes sobre sexualidad para la implementación, a posteriori, de un programa específico y orientado de intervención.

Métodos: Estudio observacional-descriptivo, cuantitativo, transversal, en una población de 250 alumnos que cursan el décimo año. Se aplicó un cuestionario con caracterización sociodemográfica y el Cuestionario de Conocimientos sobre Sexualidad (QCS), constituido por 25 preguntas de respuesta dicotómica (verdadero o falso) y organizado en seis dimensiones.

Resultados: Muestreo por conveniencia de 136 adolescentes, entre 14 y 19 años, mayormente de sexo masculino (54,4\%). El promedio de conocimiento es de 18,6 (DP=2,71) y las áreas donde los adolescentes presentan menor conocimiento son: "Primera relación sexual y relaciones sexuales", "Prevención del embarazo" y "Consejos y atención en salud sexual y reproductiva". Hay una diferencia significativa favorable para las mujeres solamente en la dimensión "Prevención del embarazo". Se identifican como factores que influyen positivamente en el conocimiento de los adolescentes la escolaridad a nivel de educación superior de los padres, con mayor enfoque en las madres, y que uno de los padres sea profesional de la salud.

Conclusión: Se constató la necesidad de desarrollar un programa de intervención orientado hacia la realidad de la escuela, con incidencia en las áreas de "Primera relación sexual y relaciones sexuales", "Prevención del embarazo" y "Consejos y atención en salud sexual y reproductiva", con orientación del programa hacia las diferencias de género y especial relevancia hacia los hombres.

\section{Introdução}

A adolescência, entendida como a transição da infância para a idade adulta, além das mudanças biológicas caraterísticas, inclui mudanças psicológicas com interferência nos relacionamentos familiares, escolares e sociais. A adolescência tem o seu início com os primeiros sinais físicos da maturidade sexual e termina com a realização social da situação de adulto independente. ${ }^{(1)}$ É comum acontecerem as primeiras relaçóes amorosas que, não sendo planeadas, muitas vezes orientam o adolescente à primeira experiência sexual. A sexualidade do adolescente é frequentemente desarmoniosa, pois a maturidade emocional nem sempre acompanha a maturidade física. (2) Há que destacar, que a sexualidade do adolescente é intrínseca e transcende o aspeto biológico, manifestando-se como um fenómeno psicológico e social, influenciado pelas crenças, valores pessoais e familiares, normas morais e tabus. ${ }^{(2)}$

A sexualidade na adolescência é considerada um problema de saúde pública, sendo a escola um local privilegiado de implementação de políticas públicas que promovam a saúde do adolescente, contribuindo para o esclarecimento e problematização relativa à educação sexual. A divulgação cada vez mais frequente nos meios de comunicação social de assuntos relacionados com o sexo, induz a precocidade da iniciação sexual e a sua banalização. ${ }^{(3,4)}$ Uma vivência sexual segura e prazerosa resulta no bem-estar emocional dos jovens, aos quais a saúde sexual e reprodutiva também diz respeito. A Organização Mundial de Saúde, recomenda uma série de metas, objetivos e pontos de ação para a Europa até 2030, que evidenciam a importância da sexualidade informada para todos e, especialmente, para os adolescentes. ${ }^{(5)}$

É responsabilidade da escola, promover a educação integral do adolescente e, naturalmente, abordar e discutir a sexualidade com vista a promoção da educação sexual. ${ }^{(3,6-8)}$ Para além de que o sucesso educacional, pode estar em risco se não acautelarmos a implementação e avaliação dos projetos de intervenção. ${ }^{(5)}$

Assim, as intervenções em meio escolar desenhadas e dirigidas com vista à modificação comportamental podem ser eficazes, viando a capacitação (empowerment) dos alunos, de forma a contribuir para a sua formação global, potenciando a adoção de estilos de vida saudáveis. ${ }^{(9)}$

A educação para a saúde deve proporcionar uma aprendizagem dos conteúdos relevantes sobre a sexualidade e levar os jovens a questionarem-se sobre as suas atitudes e decisóes que influenciam a redução de riscos. ${ }^{(2)}$

Neste contexto, surge a preocupação: quais são as necessidades e interesses dos adolescentes sobre a sexualidade? O presente estudo, tem como objetivo realizar o diagnóstico do conhecimento dos adolescentes sobre sexualidade para a implementação, à posteriori, de um programa específico e direcionado de intervenção. 


\section{Métodos}

Estudo observacional-descritivo, quantitativo e transversal, numa escola pública em zona urbana do Norte de Portugal, numa população de 250 adolescentes a frequentar o décimo ano da escolaridade obrigatória em Portugal, correspondente ao primeiro ano do Ensino Médio no Brasil.

Foram critérios de inclusão, ser aluno do décimo ano na escola em causa e, como critério de exclusão, não ter o consentimento informado assinado no dia da recolha de dados, enviado previamente para o encarregado de educação para validação.

Utilizou-se um questionário sociodemográfico para caraterização da amostra (idade, sexo, área científica que estuda, coabitação, habilitações académicas/literárias dos pais, profissão dos pais, estado civil dos pais) e o Questionário de Conhecimentos sobre Sexualidade (QCS), ${ }^{(8)}$ aplicado em abril de 2019. O QCS é constituído por 25 questôes de resposta dicotómica (verdadeiro ou falso) e organizado em seis dimensôes (D1: Primeira relação sexual e preocupaçóes sexuais; D2: Sexualidade e prazer sexual; D3: Contraceção e práticas sexuais seguras; D4: Prevenção da gravidez; D5: Infeçóes sexualmente transmissíveis e VIH/SIDA; D6: Aconselhamento e atendimento em saúde sexual e reprodutiva). Por cada resposta correta é atribuído um ponto, até ao máximo de 25 pontos. O conhecimento sobre sexualidade é maior quanto maior for a cotação no QCS, ou em determinada área.

$\mathrm{O}$ estudo atendeu às normas éticas nacionais e internacionais de pesquisas envolvendo seres humanos.

Apesar do tamanho da amostra, verificou-se a distribuição das variáveis sendo que estas não seguiram a normalidade, pelo que se recorreu a testes não paramétricos (Mann-Whitney e Kruskal-Wallis). A análise e tratamento de dados foi efetuada com o IBM SPSS Statistics Versão 25 e o nível de significância considerado foi de $\mathrm{p}<0,05$.

\section{Resultados}

A população alvo foi constituída por 250 adolescentes e, após aplicação do critério de exclusão defini- do, a amostra não probabilística fica constituída por 136 adolescentes (taxa de resposta de 54,4\%), com idades entre os 14 e 19 anos $(\mathrm{M}=15,7-\mathrm{DP}=3,3)$. $\mathrm{O}$ valor médio de conhecimentos no QCS foi de 18,6 $(\mathrm{DP}=2,71)$. As características sociodemográficas dos participantes no estudo, bem como a média, desvio padrão e testes de diferença do conhecimento sobre sexualidade (pontuação do questionário QCS), são demonstradas na tabela 1.

$\mathrm{Na}$ tabela 2, apresentam-se os dados da classificação dos conhecimentos dos adolescentes relativamente ao agrupamento de algumas variáveis como o estado civil dos pais (entre "casados/união de facto" e "outras situaçóes"), pretendendo-se perceber a influência da profissão dos pais no conhecimento dos adolescentes (entre "pelo menos um dos pais é profissional da área da saúde" e "outros"), pelo que se apresentam igualmente os dados obtidos.

Para análise do conhecimento dos adolescentes sobre a sexualidade nas seis dimensóes que o questionário permite, elaborou-se a tabela 3 , que apresenta os dados referentes à média de classificação por dimensão e os dados, por dimensão, atendendo às caraterísticas da amostra sexo e idade, e nas variáveis onde já encontramos diferença, nomeadamente: habilitaçôes literárias dos pais (ensino básico/ ensino superior); profissão dos pais (pelo menos um dos pais é profissional de saúde/nenhum dos pais é profissional de saúde).

\section{Discussão}

A adesão de pouco mais de metade dos jovens elegíveis para avaliação, não está relacionada com o facto e os jovens não quererem responder ao questionário ou participar (a perceção na altura é exatamente a oposta), mas sim pelo facto de haver uma quebra de comunicação entre alunos e encarregados de educação, não trazendo o consentimento informado assinado na data da recolha de dados para que se possa aceitar a sua participação. Segundo informações da escola onde o estudo ocorreu, é prática corrente esta falta de articulação entre alunos/diretor de turma/ encarregado de educação, sendo um aspeto que a escola está também a tentar trabalhar e melhorar. 
Tabela 1. Características sociodemográficas dos adolescentes relacionadas com o QCS $(\mathrm{n}=136)$

\begin{tabular}{|c|c|c|c|c|c|c|}
\hline \multirow{2}{*}{$\begin{array}{l}\text { Características } \\
\text { Sexo }\end{array}$} & \multicolumn{2}{|c|}{$n(\%)$} & \multicolumn{2}{|c|}{ M (DP) } & \multicolumn{2}{|c|}{$p$-value } \\
\hline & & & & & & \\
\hline Rapazes & \multicolumn{2}{|c|}{$74(54,4)$} & \multicolumn{2}{|c|}{$18,4(2,84)$} & \multicolumn{2}{|c|}{ 0,551 (b) } \\
\hline Raparigas & \multicolumn{2}{|c|}{$62(45,6)$} & \multicolumn{2}{|c|}{$18,8(2,54)$} & & \\
\hline \multicolumn{7}{|l|}{ Grupos de idade } \\
\hline$[12-14]$ anos & \multicolumn{2}{|c|}{$2(1,5)$} & \multicolumn{2}{|c|}{$18,0(2,8)$} & \multicolumn{2}{|c|}{0,744 (c) } \\
\hline$[15-17]$ anos & \multicolumn{2}{|c|}{$130(95,6)$} & \multicolumn{2}{|c|}{$18,6(2,6)$} & & \\
\hline [18-19] anos & \multicolumn{2}{|c|}{$4(2,9)$} & \multicolumn{2}{|c|}{$18,3(1,0)$} & & \\
\hline \multicolumn{7}{|l|}{ Área científica que estuda } \\
\hline Científica & \multicolumn{2}{|c|}{$67(49,3)$} & \multicolumn{2}{|c|}{$19,1(2,1)$} & & \\
\hline Humanidades & \multicolumn{2}{|c|}{$37(27,2)$} & & & & \\
\hline Artes Visuais & & & & & & \\
\hline Economico Social & & & & & & \\
\hline Curso Profissional- Informática & & & & & & \\
\hline Coabitação (mais significativos) & & & & & & \\
\hline Pais e irmãos & & & & & & \\
\hline Pais & & & & & & \\
\hline Mãe e irmão & & & & & & \\
\hline Mãe & & & & & & \\
\hline Pai & & & & & & \\
\hline Mãe e Avós & & & & & & \\
\hline Pais, irmãos e avós & & & & & & \\
\hline Pai e irmãos & & & & & & \\
\hline Mãe, irmãos e avós & & & & & & \\
\hline Mãe, irmãos e padrasto & & & & & & \\
\hline Avós & & & & & & \\
\hline Estado civil dos pais & & & & & & \\
\hline Solteiros & & & & & & \\
\hline Casados & & & & & & \\
\hline União de facto & & & & & & \\
\hline Divorciados/Separados & & & & & & \\
\hline Viúvo/a & & & & & & \\
\hline Habilitações literárias dos pais & Mãe & Pai & Mãe & Pai & Mãe & Pai \\
\hline Ensino básico e secundário & $77(56,6)$ & $84(61,8)$ & $18,1(2,7)$ & $18,4(2,7)$ & 0,037 (b) & 0,191 (b) \\
\hline Ensino Superior & $56(41,2)$ & $45(33,1)$ & $19,3(2,6)$ & $19,1(2,6)$ & & \\
\hline Sem resposta & $3(2,2)$ & $7(5,1)$ & $18,7(3,1)$ & $17,7(2,9)$ & & \\
\hline Profissão dos pais (a) & Mãe & Pai & Mãe & Pai & Mãe & Pai \\
\hline A & -- & $1(0,7)$ & $19-$ & $\therefore$ & 0,066 (c) & 0,471 (c) \\
\hline B & $12(8,8)$ & $15(11,0)$ & $19,1(2,4)$ & $18,93(2,6)$ & & \\
\hline C & $35(25,7)$ & $35(25,7)$ & $19,8(2,3)$ & $19,0(2,3)$ & & \\
\hline D & $14(10,3)$ & $9(6,6)$ & $19,0(1,8)$ & $19,4(2,1)$ & & \\
\hline E & $19(14,0)$ & $10(7,4)$ & $17,6(2,8)$ & $17,9(1,9)$ & & \\
\hline $\mathrm{F}$ & $28(20,6)$ & $18(13,2)$ & $18,1(2,8)$ & $18,0(3,3)$ & & \\
\hline G & -- & $1(0,7)$ & -- & $22,0-$ & & \\
\hline H & $2(1,5)$ & $13(9,6)$ & $19,5(0,7)$ & $17,7(2,9)$ & & \\
\hline 1 & $1(0,7)$ & $3(2,2)$ & $22,0 \quad-$ & $20,3(1,2)$ & & \\
\hline Desempregado & $15(11,0)$ & $11(8,1)$ & $17,9(3,0)$ & $19,2(2,1)$ & & \\
\hline Reformado & $1(0,7)$ & - & $18,0-$ & $\therefore$ & & \\
\hline Doméstica & $1(0,7)$ & - & 20,0 - & - & & \\
\hline Sem resposta & $8(5,9)$ & $20(14,7)$ & $16,6(3,6)$ & $17,9(3,6)$ & & \\
\hline
\end{tabular}

M - Média; DP - Desvio Padrão; a) Segundo Classificação Nacional das Profissões: A) Profissões das forças armadas; B) Representante do poder legislativo e de órgãos executivos, dirigentes, diretores e gestores executivos; C) Especialistas das atividades intelectuais e científicas; D) Técnicos e profissões de nível intermédio; E) Pessoal administrativo; F) Trabalhadores dos serviços pessoais, de proteção e segurança e vendedores; G) Agricultores e trabalhadores qualificados da agricultura, da pesca e da floresta; H) Trabalhadores qualificados da indústria, construção e artífices; I) Operadores de instalações e máquinas e trabalhadores da montagem. E acrescentou-se a situação de "Desempregado", "Reformado" e "Doméstica" por não se incluírem em nenhuma das categorias; b) Mann-Whitney; c) Kruskal-Wallis. 
Tabela 2. Resultados do QCS, conforme as variáveis agrupadas por profissão dos pais e estado civil dos pais

\begin{tabular}{|c|c|c|c|}
\hline Características & $\mathrm{n}(\%)$ & $M(D P)$ & $p$-value \\
\hline \multicolumn{4}{|l|}{ Pais com profissão na área da saúde } \\
\hline Pelo menos um dos pais é profissional de saúde & $18(13,2)$ & $20(2,2)$ & \multirow{3}{*}{0,027 (a) } \\
\hline Nenhum dos pais é profissional de saúde & $113(83,1)$ & $18,5(2,6)$ & \\
\hline Não responde & $5(3,7)$ & $16(4,2)$ & \\
\hline \multicolumn{4}{|l|}{ Estado civil dos pais } \\
\hline Casado /União de facto & $75(55,2)$ & $18,3(0,32)$ & \multirow{3}{*}{0,345 (a) } \\
\hline Outra situação & $60(44,1)$ & $18,9(0,34)$ & \\
\hline Não responde & $1(0,7)$ & $16,6-$ & \\
\hline
\end{tabular}

(a) Kruskal-Wallis; M - Média; DP - Desvio Padrão

Tabela 3. Distribuição do conhecimento dos adolescentes por dimensão em função do sexo, grupos de idades, habilitações literárias da mãe e do pai e na situação de pelo menos um pai ser profissional da área da saúde

\begin{tabular}{|c|c|c|c|c|c|c|}
\hline \multirow[t]{2}{*}{ Características } & \multicolumn{6}{|c|}{ Dimensões do QCS (Média/DP) } \\
\hline & D1 & D2 & D3 & D4 & D5 & D6 \\
\hline Classificação total & $2,72(0,90)$ & $2,80(0,49)$ & $4,52(0,97)$ & $1,25(0,69)$ & $5,00(1,27)$ & $1,27(0,56)$ \\
\hline \multicolumn{7}{|l|}{ Sexo } \\
\hline Rapazes & $2,82(0,90)$ & $2,74(, 58)$ & 4,55 (,92) & $1,12(, 62)$ & $5,11(1,31)$ & $1,26(, 55)$ \\
\hline Raparigas & $2,60(, 90)$ & $2,87(, 38)$ & $4,48(1,04)$ & $1,40(, 74)$ & $4,87(1,22)$ & $1,29(, 58)$ \\
\hline$P(b)$ & 0,143 & 0,125 & 0,677 & 0,018 & 0,280 & 0,731 \\
\hline \multicolumn{7}{|l|}{ Grupos de idade } \\
\hline$[12-14]$ anos & $3,50(0,71)$ & $2,50(0,71)$ & $5,50(0,71)$ & $1,00(0,00)$ & $4,50(0,71)$ & $2,00(0,00)$ \\
\hline [15-17] anos & $2,72(0,91)$ & $2,82(0,48)$ & $4,51(0,97)$ & $1,25(0,69)$ & $5,10(1,29)$ & $1,26(0,57)$ \\
\hline [18-19] anos & $2,25(0,50)$ & $2,50(1,0)$ & $4,50(1,29)$ & $1,50(0,58)$ & $5,00(0,82)$ & $1,25(0,50)$ \\
\hline$P(b)$ & 0,194 & 0,347 & 0,301 & 0,634 & 0,656 & 0,158 \\
\hline \multicolumn{7}{|l|}{ Habilitações literárias da Mãe } \\
\hline Ensino básico e secundário & $2,64(0,87)$ & $2,79(0,27)$ & $4,38(1,00)$ & $1,19(0,50)$ & $4,87(1,39)$ & $1,18(0,53)$ \\
\hline Ensino Superior & $2,86(0,94)$ & $2,80(0,23)$ & $4,73(0,82)$ & $1,32(0,64)$ & $5,18(1,09)$ & $1,39(0,59)$ \\
\hline Sem resposta & $2,33(0,58)$ & $3,0(0,00)$ & $4,33(1,16)$ & $1,33(1,16)$ & $5,00(1,00)$ & $1,33(0,58)$ \\
\hline$p(b)$ & 0,214 & 0,758 & 0,101 & 0,584 & 0,543 & 0,081 \\
\hline \multicolumn{7}{|l|}{ Habilitações literárias do Pai } \\
\hline Ensino básico e secundário & $2,68(0,93)$ & $2,77(0,55)$ & $4,48(0,94)$ & $1,26(0,68)$ & $4,88(1,31)$ & $1,27(0,59)$ \\
\hline Ensino Superior & $2,80(0,89)$ & $2,82(0,44)$ & $4,58(0,99)$ & $1,24(0,68)$ & $5,24(1,15)$ & $1,31(0,51)$ \\
\hline Sem resposta & $2,71(4,88)$ & $3,0(0,00)$ & $4,71(1,38)$ & $1,14(0,90)$ & $4,86(1,46)$ & $1,0(0,58)$ \\
\hline$p(b)$ & 0,641 & 0,499 & 0,522 & 0,950 & 0,368 & 0,432 \\
\hline \multicolumn{7}{|l|}{ Pais com profissão na área da saúde } \\
\hline Pelo menos um dos pais é profissional de saúde & $2,83(0,71)$ & $2,89(0,32)$ & $4,78(0,94)$ & $1,56(0,51)$ & $5,40(1,19)$ & $1,28(0,58)$ \\
\hline Nenhum dos pais é profissional de saúde & $2,73(0,94)$ & $2,81(0,49)$ & $4,50(0,97)$ & $1,20(0,69)$ & $4,90(1,25)$ & $1,26(0,56)$ \\
\hline Não responde & $2,20(0,45)$ & $2,4(0,89)$ & $4,0(1,23)$ & $1,20(0,84)$ & $4,0(1,58)$ & $1,6(0,55)$ \\
\hline$p(b)$ & 0,232 & 0,239 & 0,320 & 0,146 & 0,100 & 0,403 \\
\hline
\end{tabular}

DP - Desvio Padrão $\quad$ (b) Kruskal-Wallis

D1 - Primeira relação sexual e preocupações sexuais (pontuação máxima: 5 pontos)

D2 - Sexualidade e prazer sexual (pontuação máxima: 3 pontos)

D3 - Contraceção e práticas sexuais seguras (pontuação máxima: 6 pontos)

D4 - Prevenção da gravidez (pontuação máxima: 2 pontos)

D5 - Infeções sexualmente transmissiveis e VIH/SIDA (pontuação máxima: 7 pontos)

D6 - Aconselhamento e atendimento em saúde sexual e reprodutiva (pontuação máxima: 2 pontos)

Existe alguma limitação da representatividade do estudo, no entanto, a sua reprodutibilidade fácil poderá ser uma mais-valia para outros locais com características semelhantes.

À semelhança de outros estudos, há mais rapazes do que raparigas e são as raparigas que apresentam maiores conhecimentos sobre sexualidade, mas sem diferença significativa. ${ }^{(9)}$ No entanto outros estudos encontraram esta diferença como relevante, ${ }^{(9)}$ demonstrando haver necessidade de dirigir os progra- mas de intervenção para os rapazes e ir ao encontro da sua especificidade.

O valor médio de conhecimento no questionário QCS é de 18,6 pontos num máximo de 25 possíveis, sendo um valor ligeiramente superior ao de outros estudos. ${ }^{(9)}$

Relativamente à variável idade, o conhecimento sobre sexualidade parece aumentar com esta ${ }^{(9)}$ e, neste estudo, constata-se que os alunos que têm mais conhecimentos são os adolescentes mais velhos. 
Verifica-se uma maior adesão à resposta ao questionário por parte dos alunos da área científica, sendo também estes alunos que apresentam melhores conhecimentos na área da sexualidade, muito provavelmente por estarem mais sensibilizados para a temática, dado esta fazer parte dos conteúdos programático escolares.

A grande maioria vive com os pais, mas não é a presença destes que implica mais conhecimentos na área da sexualidade, já que sempre que há presença de outras pessoas, avós e padrasto, encontramos valores de conhecimento sobre sexualidade mais elevados. Por outro lado, as famílias onde o pai é o único progenitor presente, evidenciam menores conhecimentos sobre sexualidade, fruto da sua postura mais fechada, menos participativa e menos permissiva do que as mães, influenciando diretamente a vida dos adolescentes. ${ }^{(10)}$

O facto de os pais estarem solteiros ou divorciados/separados parece influenciar positivamente o conhecimento sobre sexualidade, o que pode estar relacionado com o facto de ser nestas famílias que, naturalmente, coabitam vários elementos diferentes dos das famílias clássicas (padrasto, outras pessoas), tal como evidenciado na coabitação.

A família é naturalmente o principal meio para aquisição de valores para viver em sociedade, mas esta poderá não corresponder nem às espectativas nem às necessidades dos jovens, recorrendo a outras fontes. É evidente a importância da educação informal para a educação dos adolescentes e o papel decisivo dos profissionais da área da saúde escolar, respondendo aos pressupostos necessários. ${ }^{(5,10)}$

As habilitaçôes literárias influenciam o conhecimento dos adolescentes sobre sexualidade, sendo este maior quanto maior for a escolaridade dos pais, tal como noutros estudos. ${ }^{(9)}$ Também são os adolescentes filhos de mães com ensino superior que apresentam melhores conhecimentos, e aqui com diferença estatística significativa.

A profissão dos pais influencia o conhecimento sobre sexualidade, sendo as profissões que requerem uma escolaridade ao nível do ensino superior para as exercer, aquelas em que os adolescentes demonstram mais conhecimentos. Já os adolescentes que têm pelo menos um dos pais na área da saúde apre- sentam melhores conhecimentos na área da sexualidade e com diferença estatística significativa.

Relativamente às seis dimensões que o questionário avalia, aquelas áreas em que os adolescentes apresentam piores conhecimentos são D1 - Primeira relação sexual e preocupaçóes sexuais e D4 - Prevenção da gravidez, aqui com as raparigas a apresentarem melhores conhecimentos nesta área (com diferença estatística significativa), muito provavelmente pela preocupação na temática já que, socialmente, a gravidez ainda incorre em consequências mais diretas e imediatas para as raparigas e naturalmente a procura da pílula do dia seguinte ou o aborto. ${ }^{(11)}$

Ainda em relação às dimensôes, não há diferenças relevantes no conhecimento nas diversas dimensôes em relação às idades, habilitações literárias da mãe ou do pai e o facto de o adolescente ter pelo menos um dos pais na área da saúde.

Assim, a metodologia aqui explorada e o questionário simples utilizado (QCS), tem a potencialidade de facilmente ser utilizado pelas instituiçôes de ensino ou outras, que pretendam um diagnóstico rápido que fundamente a planificação de intervençôes para prevenção de comportamentos de risco na área da sexualidade, indo ao encontro do preconizado pela OMS. ${ }^{(5)} \mathrm{O}$ conhecimento não indica que haverá obrigatoriamente mudanças nos comportamentos, no entanto, predispóe para uma conduta auto protetora e minimizadora de riscos..$^{(2,5)}$

\section{Conclusão}

Do diagnóstico realizado evidenciam-se menores conhecimentos nas áreas de "primeira relação sexual e relaçóes sexuais", "prevenção da gravidez" e "aconselhamento e atendimento em saúde sexual e reprodutiva", pelo que recomendamos a intervenção dirigida nestas áreas, com especial ênfase nas diferenças entre rapazes e raparigas.

\section{Colaborações}

Silva SMDT, Ferreira MMSV, Amaral-Bastos MM, Monteiro MAJ e Couto GR declararam que par- 
ticiparam da concepção do estudo, análise e interpretação dos dados, redação do manuscrito, revisão crítica do conteúdo e aprovação da versão final a ser publicada.

\section{Referências}

1. Margarida GM, Reis M, Ramiro L, Pais RJ, Leal I. [Sexual education in Portugal: Review of legislation and implementation in schools]. Psicol Saúde Doenças. 2014;15(2):335-55. Portuguese.

2. Kerntopf MR, Lacerda JF, Fonseca NH, Nascimento EP, Lemos IC, Fernandes GP, et al. [Sexuality in adolescence: a critical literature review]. Adolesc Saude. 2016; 13(2):106-13. Portuguese.

3. Oliveira AC, Caramelo F, Patrício M, Camarneiro AP, Cardoso SM, Pita JR. [Impact of an educational intervention program on the sexual behaviors of higher education students]. Rev Enferm Ref. 2017;13:7182. Portuguese.

4. Nelas P, Coutinho E, Chaves C, Amaral O, Cruz C. [Knowledge about family planning in higher education students]. International J Develop Educ Psychol. 2018;2(1):187-96. Portuguese.

5. World Health Organization (WHO). Regional Office for Europe. Action Plan for Sexual and Reproductive Health: Towards achieving the 2030 Agenda for Sustainable Development in Europe - leaving no one behind]
[Internet]. Copenhagen: WHO; 2016. [cited 2019 Jun 10]. Available from: http://www.euro.who.int/_data/assets/pdf_file/0003/322275/ Action-plan-sexual-reproductive-health.pdf?ua=1.

6. Brasil, Leis, Decretos. Regime de Aplicação da Educação Sexual em Meio Escolar. Lei n. 60 de 6 de agosto de 2009. Diário da República [Internet]. 2009 [citado 2019 Feb 10]; 1. ${ }^{a}$ série, N. ${ }^{0} 151$ A: 5097 5098. Disponivel em: https://data.dre.pt/eli/lei/60/2009/08/06/p/dre/ $\mathrm{pt} / \mathrm{html}$. Portuguese.

7. Portaria no. 196 $/ 2010$. Regulamenta a Lei n. 60 de 6 de agosto de 2009, que estabelece o regime de aplicação da educação sexual em meio escolar. Portaria n. ${ }^{\circ} 196-A,{ }^{\circ} .{ }^{\circ} 69$, de 9 de abril de 2010. Diário da República [Internet]. 2010 [citado 2019 Feb 10]; 1. ${ }^{\text {a }}$ série. p. 1170(2)-1170(4). Disponível em: https://data.dre.pt/eli/port/196a/2010/04/09/p/dre/pt/html.

8. Matos MG, Reis M, Ramiro L, Pais Ribeiro J, Leal I. [Sexual education in Portugal: Review of legislation and implementation in schools]. Psicol Saúde Doenças. 2014; 15(2):335-55. Portuguese.

9. Carvalho CP, Pinheiro MR, Gouveia JP, Vilar DR. [Knowledge about sexuality: Construction and validation of an assessment instrument for adolescents]. Rev Portug Educ. 2017; 30(2):249-74. Portuguese.

10. Nery IS, Freitosa JJ, Sousa AF, Fernandes AC. Approach to sexuality in the dialogue between parents and adolescents. Acta Paul Enferm. 2015;28(3):287-92.

11. Li C, Cheng Z, Wu T, Liang X, Gaoshan J, Li I, et al. The relationships of school-based sexuality education, sexual knowledge and sexual behaviors - a study of 18,000 Chinese college students. Reprod Health. 2017; $14: 103$. 